\title{
Nonalcoholic fatty liver disease in chronic obstructive pulmonary disease
}

\author{
Damien Viglino ${ }^{1,2,9}$, Ingrid Jullian-Desayes ${ }^{1,3,9}$, Mélanie Minoves ${ }^{1,3}$, \\ Judith Aron-Wisnewsky ${ }^{4,5,6}$, Vincent Leroy ${ }^{7,8}$, Jean-Pierre Zarski ${ }^{7,8}$, \\ Renaud Tamisier ${ }^{1,3}$, Marie Joyeux-Faure ${ }^{1,3,10}$ and Jean-Louis Pépin ${ }^{1,3,10}$
}

Affiliations: ${ }^{1} \mathrm{HP} 2$ laboratory, INSERM U1042, Grenoble Alps University, Grenoble, France. ${ }^{2}$ Emergency Dept and Mobile Intensive Care Unit, Grenoble Alps University Hospital, Grenoble, France. ${ }^{3} \mathrm{EFCR}$ Laboratory, Grenoble Alps University Hospital, Grenoble, France. ${ }^{4}$ Institute of Cardiometabolism and Nutrition, ICAN, Assistance Publique Hôpitaux de Paris, Pitié-Salpêtrière Hospital, Paris, France. ${ }^{5}$ Nutriomics Team, UMR_S 1166, INSERM, Paris, France. ${ }^{6}$ Nutriomics Team UMR_S 1166, UPMC University Paris 06, Sorbonne Universités, Paris, France. ${ }^{7}$ Hepatogastroenterology Dept, Digidune, Grenoble Alps University Hospital, Grenoble, France. ${ }^{8}$ INSERM U823, IAPC Institute for Advanced Biosciences, University Grenoble Alps, Grenoble, France. ${ }^{9}$ Both authors contributed equally. ${ }^{10}$ Both authors contributed equally.

Correspondence: Jean-Louis Pépin, CHU Grenoble CS 1021738043 Grenoble Cedex 9, France.

E-mail: JPepindachu-grenoble.fr

@ERSpublications

Nonalcoholic fatty liver disease is highly prevalent in COPD patients and may contribute to comorbidities http://ow.ly/cH2h309yOlM

Cite this article as: Viglino D, Jullian-Desayes I, Minoves M, et al. Nonalcoholic fatty liver disease in chronic obstructive pulmonary disease. Eur Respir J 2017; 49: 1601923 [https://doi.org/10.1183/ 13993003.01923-2016].

ABSTRACT Nonalcoholic fatty liver disease (NAFLD) is independently linked to cardiometabolic morbidity and mortality. Low-grade inflammation, oxidative stress and ectopic fat, common features of chronic obstructive pulmonary disease (COPD), might contribute to the development of NAFLD.

We aimed to investigate the prevalence of NAFLD and to evaluate the relationship between various types of liver damage and COPD severity, comorbidities and circulating inflammatory cytokines. Validated noninvasive tests (FibroMax: SteatoTest, NashTest and FibroTest) were used to assess steatosis, nonalcoholic steatohepatitis (NASH) and liver fibrosis. Patients underwent an objective assessment of COPD comorbidities, including sleep studies. Biological parameters included a complete lipid profile and inflammatory markers.

In COPD patients the prevalence of steatosis, NASH and fibrosis were $41.4 \%, 36.9 \%$ and $61.3 \%$, respectively. In multivariate analysis, SteatoTest and FibroTest were significantly associated with sex, body mass index (BMI), untreated sleep apnoea and insulin resistance, and, in addition, COPD Global Initiative for Chronic Obstructive Lung Disease stage for SteatoTest. Patients with steatosis had higher tumour necrosis factor- $\alpha$ levels and those with NASH or a combination of liver damage types had raised leptin levels after adjustment for age, sex and BMI.

We concluded that NAFLD is highly prevalent in COPD and might contribute to cardiometabolic comorbidities.

This article has supplementary material available from erj.ersjournals.com

Received: Sept 302016 | Accepted after revision: Feb 232017

Support statement: "Air Liquide” Foundation, endowment fund “AGIR pour les Maladies Chroniques", and "Mutualia" provided unrestricted funding for the management of the cohort. This research was conducted with support from AstraZeneca. This work was also supported by the French National Research Agency in the framework of the "Investissements d'avenir" programme (ANR-15-IDEX-02). Funding information for this article has been deposited with the Crossref Funder Registry.

Conflict of interest: None declared.

Copyright CERS 2017 


\section{Introduction}

Nonalcoholic fatty liver disease (NAFLD) is a worldwide pandemic with $25 \%$ prevalence $[1,2]$. NAFLD is characterised by hepatic triglyceride accumulation $(>5 \%)$, known as steatosis, and the absence of significant alcohol consumption or secondary causes, mainly viral hepatitis [3]. While some patients remain at the stage of steatosis with or without mild inflammation [4], others progress to nonalcoholic steatohepatitis (NASH), defined as the presence of steatosis, hepatocyte ballooning and inflammatory infiltration $[4,5]$. NASH may be accompanied by liver fibrosis, which accounts for prognosis [6,7], which can further progress towards cirrhosis and hepatocarcinoma $[8,9]$. NAFLD has a complex and two-way relationship with metabolic syndrome. Not only is there a cross-sectional association between the metabolic syndrome and NAFLD, but NAFLD may be a precursor of metabolic syndrome [10-12]. It has been reported that NAFLD diagnosis almost doubles the risk of incident metabolic syndrome in the next few years [12]. NAFLD combines with metabolic comorbidities such as obesity, type 2 diabetes, hyperlipidaemia and hypertension, leading to a two-fold increase in liver-specific and overall mortality [2]. In addition, recent studies suggest that NAFLD may be considered as a novel independent cardiovascular risk factor [13].

Metabolic syndrome is common in chronic obstructive pulmonary disease (COPD) patients [14, 15] with a prevalence ranging from $23 \%$ to $53 \%$, mainly depending upon Global Initiative for Chronic Obstructive Lung Disease (GOLD) grades [16-18] and the patient's inflammatory status [19-21]. COPD is strongly associated with major cardiovascular risk factors and COPD patients exhibit a two to five times higher risk of ischaemic heart disease, cardiac arrhythmias or heart failure [22]. There is increasing evidence that inflammatory visceral fat depots are significant contributors to systemic inflammation and COPD-related cardiometabolic comorbidities [19, 23, 24]. Interestingly, the literature reports a higher prevalence of all-cause liver disease in patients with COPD, but only a small number of patients with NAFLD [25]. Therefore, the specific links between NAFLD and COPD need to be confirmed and further evaluated.

NAFLD has been poorly studied in COPD patients, although there is a strong mechanistic rationale supporting the hypothesis of increased NAFLD prevalence in COPD patients. There is still debate over the "spill-over" theory: that oxidative stress and systemic inflammation reported in some COPD subtypes might participate in the production of reactive oxygen species and inflammation in the liver. Patients with COPD have increased visceral fat, a known inflammatory and lipolitic fat deposit [24, 26]. Thus, the subsequent increase in free fatty acids accumulating in the liver might lead to NAFLD development in COPD. Nocturnal hypoxia subsequent to COPD per se, or related to the obstructive sleep apnoea (OSA) that is frequently associated with it, might also trigger the development of NAFLD [9, 27, 28].

FibroMax is a noninvasive test for NAFLD screening that has been validated against liver biopsies [29] and is proposed by European guidelines [7]; the algorithm uses the association of sex, age, weight, height and numerous serum biomarkers. FibroMax uses SteatoTest, NashTest and FibroTest for the noninvasive evaluation of liver steatosis, NASH and liver fibrosis, respectively. The aims of our study were 1) to use noninvasive blood tests (FibroMax) to evaluate the prevalence of steatosis, NASH and fibrosis in a well phenotyped cohort of COPD patients; and 2) to evaluate the relationship between steatosis, NASH and fibrosis, and COPD severity, comorbidities and circulating inflammatory cytokines.

\section{Methods}

Ethics and consent

Patients included in this study were part of two ongoing COPD cohorts attending Grenoble University Hospital (Grenoble, France). The studies were conducted in accordance with applicable good clinical practice requirements in Europe, French law, International Council for Harmonisation E6 recommendations and the ethical principles of the Declaration of Helsinki. These studies were approved by an independent ethics committee (Comité de Protection des Personnes, Grenoble IRB0006705). The possibility of later analysis of patient blood samples, including exploration of their inflammatory status and hepatic features (this ancillary study) was initially mentioned in the protocol and was approved and authorised. These studies have been registered with ClinicalTrials.gov (NCT00404430 and NCT01195064).

\section{Patients}

The vast majority of patients were recruited during outpatient consultations. Inpatients referred for an acute exacerbation of COPD $(n=7,6 \%)$ represented a small percentage of the study population. Participation in the prospective cohort was proposed to consecutive patients, but the rate of refusal was high, owing to complex phenotyping requiring additional blood samples, cardiovascular phenotyping and in-lab polysomnography. Patients aged $>18$ years presenting with mild to severe COPD were eligible. We excluded patients with cancer, daily alcohol consumption $\geqslant 20 \mathrm{~g}$ for females and $\geqslant 30 \mathrm{~g}$ for males or viral hepatitis. All patients with a positive NashTest (stage $\geqslant \mathrm{N} 1$, see the procedures section) were checked to 
exclude secondary hepatic fat accumulation owing to significant alcohol consumption, use of steatogenic medication or hereditary disorders [1].

\section{Procedures}

Comorbidities were assessed based on predefined cut-offs [19] with objective clinical measurements, laboratory analyses (see later) and a systematic sleep study.

At study enrolment, fasting serum samples were collected from all patients, frozen and stored at $-80^{\circ} \mathrm{C}$ until analyses were conducted.

\section{Metabolic parameters}

10 biochemical markers, including liver enzymes, lipid profile, fasting glycaemia and insulin were measured in order to characterise metabolic comorbidities and calculate FibroMax scores: $\alpha 2$-macroglobulin, apolipoprotein A1, haptoglobin, $\gamma$-glutamyltransferase (GGT), total bilirubin, alanine aminotransferase (ALT), aspartate aminotransferase (AST), fasting blood glucose, triglycerides and total cholesterol. Anonymised data were sent to the calculation centre (BioPredictive, Paris, France) to obtain FibroMax scores blinded to the severity of COPD and comorbidities. Insulin resistance was assessed using the homeostatic model assessment for insulin resistance (HOMA-IR), calculated using the following formula: insulinaemia $\times$ glucose/22.5 (glucose units $\mathrm{mmol} \cdot \mathrm{L}^{-1}$ ) [30].

\section{FibroMax test}

NAFLD screening using ultrasonography [31], together with the exclusion of competing aetiologies [3, 32] is recommended in certain groups at high risk [33]. In this clinical study, prevalence was based on noninvasive FibroMax. FibroMax combines three tests: FibroTest, SteatoTest and NashTest [29, 34]. FibroTest includes $\alpha 2$-macroglobulin, apolipoprotein A1, haptoglobin, GGT and total bilirubin values, age and sex. It provides a quantitative estimate ranging from 0.00 to 1.00 , with higher values corresponding to greater probability of lesions. Scores correspond to a METAVIR stage [35] as follows: F0 (0.00-0.21); F0-F1 (0.22-0.27); F1 (0.28-0.31); F1-F2 (0.32-0.48); F2 (0.49-0.58); F3 (0.59-0.72); F3-F4 (0.73-0.74) and F4 $(0.75-1.0)$ [34]. The test gives an area under the receiver operating characteristic curve (AUC) of 0.89 [29]. This value appears to be better for the diagnosis of advanced fibrosis and cirrhosis than the validated NAFLD fibrosis score (AUC 0.76) [29]. SteatoTest combines FibroTest parameters with height and weight plus ALT, serum fasting glucose, triglycerides and cholesterol. SteatoTest scores range from 0.00 to 1.00 , determining four steatosis stages from S0 to S4, as follows. S0 (0.00-0.37): no steatosis; S1 $(0.38-0.56)$ : minimal steatosis, <5\%; S2 (0.57-0.68): moderate steatosis, 6-32\%; and S3-S4 (0.69-1.0): severe steatosis, $>32 \%$. SteatoTest appears to be better $(\mathrm{AUC}=0.80)$ for the diagnosis of steatosis than conventional markers such as GGT or ALT [29]. Finally, the NashTest score is calculated from weight, height, AST, serum fasting glucose, triglycerides and cholesterol in addition to age, sex and FibroTest components. Thus, the NashTest distinguishes three NASH categories: N0 (0.00-0.25) for absence of NASH; N1 $(>0.25-0.50)$ for borderline NASH; and N2 (>0.50-0.75) for NASH $[29,34]$. The cut-off values of the NashTest for the diagnosis of NASH appear appropriate for our cohort (AUC 0.80) [29]. FibroTest $\geqslant$ F0-F1, SteatoTest $\geqslant S 2$ and NashTest $\geqslant N 1$ were considered positives, as previously applied by MinvilLe et al. [27]. This choice is justified by the fact that any degree of liver fibrosis and/or inflammation is considered a significant pathological condition, while mild steatosis has usually little impact on liver prognosis. When at least two types of liver damage were associated, the patient was assigned to a "combination of liver diseases" group.

\section{Inflammatory markers}

Serum levels of adiponectin, leptin, resistin and tumour necrosis factor (TNF)- $\alpha$ were measured using multiplex assays. Interleukin (IL)-6 was measured using immunoassay and ultrasensitive C-reactive protein (usCRP) using an automated immunonephelometry technique.

\section{Data analysis}

Continuous data are presented as mean \pm SD and categorical data as frequency and percentage.

The prevalence of positive FibroMax tests is expressed as percentage. The relationship (odds ratio) between SteatoTest or NashTest and phenotyping characteristics was analysed by logistic regression. The relationship between FibroTest and phenotyping characteristics was analysed by linear regression. In univariate logistic regression, when a continuous variable was not log-linear, a new variable was created from the quartiles or the median value. Independent parameters were included in the multivariate model when significance was $\leqslant 0.05$ in the univariate model. Inflammatory markers were not included in multivariate analyses, but were investigated as exploratory variables to gain pathophysiological insights. 
The inflammatory parameters are presented as boxplots between patients without liver disease and patients with a positive SteatoTest, positive NashTest, positive FibroTest or combined liver diseases.

All statistical analyses were performed using SAS 9.4 software (SAS Institute, Cary, NC, USA).

\section{Results}

Patient characteristics

Of the 132 eligible COPD patients, 21 were excluded due to their daily alcohol consumption ( $\geqslant 20 \mathrm{~g}$ for females and $\geqslant 30 \mathrm{~g}$ for males). As a result, $111 \mathrm{COPD}$ patients with available serum samples were included. Patient characteristics are given in table 1 . The included patients were predominantly male (77.5\%), mean age was $63.8 \pm 8.7$ years and mean body mass index (BMI) was $25.1 \pm 4.5 \mathrm{~kg} \cdot \mathrm{m}^{-2} \cdot 74.8 \%$ of patients had GOLD grade $1-2$ and $25.2 \%$ had GOLD grade III-IV COPD).

\section{Prevalence and risk factors of liver disease in COPD}

Stages of FibroTest, SteatoTest and NashTest in the entire COPD population are reported in the supplementary table S1. The prevalence of liver disease was $41.4 \%$ of moderate to severe steatosis (stages $\geqslant \mathrm{S} 2$ ), $36.9 \%$ of borderline NASH and NASH (stages $\geqslant \mathrm{N} 1$ ) and $61.3 \%$ of fibrosis (stages $\geqslant \mathrm{F} 0-\mathrm{F} 1$ ) and is presented according to COPD GOLD grade in table 2. The prevalence of a combination of different types of liver damage is reported in online supplementary table S1 and we observed that $17 \%$ of patients fulfilled the criteria of all three studied abnormalities. Clinical characteristics and comorbidities of subjects without liver abnormalities, or with steatosis, NASH and fibrosis individually and combined are detailed in online supplementary table S5.

\begin{tabular}{|c|c|}
\hline Patients n & 111 \\
\hline Age years & $63.8 \pm 8.7$ \\
\hline Male & 77.5 \\
\hline$B M I \mathbf{k g} \cdot \mathrm{m}^{-2}$ & $25.1 \pm 4.5$ \\
\hline \multicolumn{2}{|l|}{ History } \\
\hline \multicolumn{2}{|l|}{ Smoking } \\
\hline Current & 33.3 \\
\hline Ex-smokers & 65.8 \\
\hline Never-smokers & 0.9 \\
\hline \multicolumn{2}{|l|}{ OSA } \\
\hline Untreated & 34.3 \\
\hline Treated & 9.3 \\
\hline Type 2 diabetes & 15.3 \\
\hline Hypertension & 47.7 \\
\hline Dyslipidaemia & 40.5 \\
\hline \multicolumn{2}{|l|}{ Biological parameters } \\
\hline Fasting glucose $\mathrm{mmol} \cdot \mathrm{L}^{-1}$ & $5.7 \pm 2.0$ \\
\hline Insulin $\mu \mathrm{IU} \cdot \mathrm{mL}^{-1}$ & $13.1 \pm 14.2$ \\
\hline HOMA-IR & $3.6 \pm 4.5$ \\
\hline AST U.L $L^{-1}$ & $24.1 \pm 19.9$ \\
\hline$A L T U \cdot L^{-1}$ & $33.9 \pm 19.8$ \\
\hline GGT U.L $L^{-1}$ & $87.6 \pm 65.4$ \\
\hline \multicolumn{2}{|l|}{ Pulmonary function tests } \\
\hline Post-bronchodilator FEV1 \% & $64.4 \pm 20.8$ \\
\hline Post-bronchodilator FVC \% & $92.6 \pm 19.6$ \\
\hline $\mathrm{FEV}_{1} / \mathrm{FVC} \%$ & $55.1 \pm 13.3$ \\
\hline \multicolumn{2}{|l|}{ GOLD grade } \\
\hline I & 15.3 \\
\hline II & 59.5 \\
\hline III & 18.9 \\
\hline IV & 6.3 \\
\hline
\end{tabular}

Data are presented as mean \pm SD or $\%$, unless otherwise stated. BMI: body mass index; OSA: obstructive sleep apnoea; HOMA-IR: homeostasis model assessment for insulin resistance; AST: aspartate aminotransferase; ALT: alanine aminotransferase; GGT: $\gamma$-glutamyltransferase; FEV1: forced expiratory volume in 1 s; FVC: forced vital capacity; GOLD: Global Initiative for Chronic Obstructive Lung Disease. 
TABLE 2 Prevalence of positive SteatoTest, NashTest and FibroTest in the chronic obstructive pulmonary disease (COPD) population, based on Global Initiative for Chronic Obstructive Lung Disease (GOLD) grades

\begin{tabular}{lccccc} 
& \multicolumn{3}{c}{ COPD GOLD grades } & Total & p-value \\
\cline { 2 - 4 } & I & II & III-IV & & \\
\hline Patients n & 17 & 66 & 28 & 111 & \\
Positive SteatoTest & $2(11.8)$ & $31(47.0)$ & $13(46.4)$ & $46(41.4)$ & $\mathbf{0 . 0 2 6}$ \\
Positive NashTest & $5(29.4)$ & $25(37.9)$ & $11(39.3)$ & $41(36.9)$ & 0.777 \\
Positive FibroTest & $8(47.1)$ & $44(66.7)$ & $16(57.1)$ & $68(61.3)$ & 0.293
\end{tabular}

Data are presented as $\mathrm{n}(\%)$, unless otherwise stated. Data presented in bold type are statistically significant.

\section{Steatosis}

In univariate analysis, steatosis was significantly associated with male sex, high BMI, current smoking, untreated OSA, dyslipidaemia, insulin resistance (expressed as HOMA-IR score) and post-bronchodilator forced vital capacity (FVC) (online supplementary table S2). In multivariate analysis, male sex, high BMI, untreated OSA, HOMA-IR and COPD severity were independent risk factors (table 3).

\section{NASH}

Age, BMI, current smoking, dyslipidaemia and post-bronchodilator FVC were linked with a positive NashTest in univariate analysis (online supplementary table S3). In multivariate analysis, only BMI was a risk factor for NASH (table 3 ).

\section{Fibrosis}

In univariate analysis, the various degrees of fibrosis severity were significantly associated with age, insulin resistance and untreated OSA. Female, nondiabetic and nonhypertensive patients were less at risk of developing fibrosis (online supplementary table S4). In multivariate analysis, age, male sex, untreated OSA and insulin resistance (HOMA-IR) were risk factors for having a positive FibroTest (table 3).

\section{Adipokines, inflammatory status and liver disease in COPD}

Levels of adipokines (leptin, adiponectin and resistin) and inflammatory markers (TNF- $\alpha$, IL- 6 and usCRP) in five groups (no liver disease, steatosis, NASH, fibrosis and a combination of different types of

\section{TABLE 3 Multivariate analysis of the three tests included in FibroMax}

\begin{tabular}{lcc} 
& Odds ratio (95\% Cl) & p-value \\
\hline SteatoTest & & \\
Sex (male versus female) & $5.62(1.05-30.07)$ & $\mathbf{0 . 0 4 4}$ \\
BMI & $1.44(1.20-1.74)$ & $\mathbf{0 . 0 0 0 1}$ \\
Untreated OSA versus non-OSA & $6.84(1.68-27.77)$ & $\mathbf{0 . 0 0 7}$ \\
HOMA-IR & $1.25(1.02-1.55)$ & $\mathbf{0 . 0 3 6}$ \\
GOLD stage II versus I & $21.80(2.36-201.02)$ & $\mathbf{0 . 0 0 7}$ \\
GOLD stage III-IV versus I & $26.77(2.34-306.29)$ & $\mathbf{0 . 0 0 8}$ \\
NashTest & & 0.230 \\
Age & $1.05(0.97-1.13)$ & $<\mathbf{0 . 0 0 0 1}$ \\
BMI & $1.40(1.18-1.66)$ & 0.274 \\
Current smoker versus ex- or never-smokers & $0.49(0.14-1.75)$ & 0.286 \\
Dyslipidaemia & $1.72(0.64-4.62)$ & 0.568 \\
Post-bronchodilator FVC \% & $0.99(0.97-1.02)$ & $\mathbf{0 . 0 2 0}$ \\
FibroTest & & $\mathbf{0 . 0 1 0}$ \\
Age & $0.005(0.001-0.009)$ & $\mathbf{0 . 0 3 0}$ \\
Sex (male versus female) & $0.11(0.02-0.20)$ & $\mathbf{0 . 0 0 0 2}$ \\
Untreated OSA versus non-OSA & $0.08(0.01-0.15)$ & \\
HOMA-IR & $0.02(0.01-0.02)$ & \\
\hline
\end{tabular}


liver damage) are shown in figures 1 and 2 and in online supplementary table S5. Patients with steatosis had higher TNF- $\alpha$ levels than patients with no liver disease $(p=0.016)$ after adjustment for age, sex and BMI. COPD patients with a combination of histological liver alterations had higher leptin levels compared to those with no liver damage $(p=0.047)$ after adjustment for age, sex and BMI. Finally, after the same adjustments, patients with a positive NashTest had significantly higher leptin levels than patients without liver damage $(\mathrm{p}=0.039)$.

\section{Discussion}

To our knowledge, this study is the first to investigate the prevalence and severity of NAFLD using noninvasive blood tests in a relatively large group of well-characterised COPD patients. The prevalence in COPD patients of steatosis, NASH and fibrosis was $41 \%, 37 \%$ and $61 \%$, respectively. Liver steatosis, NASH and fibrosis were primarily linked with the metabolic comorbidities of COPD (i.e. obesity and insulin resistance) and systemic inflammation.

There is an increase in awareness regarding the various COPD clinical subtypes $[19,36]$. The concomitant chronic diseases and systemic comorbidities of COPD represent a major challenge for risk prediction and complicate integrated care delivery. Clusters of comorbidities, essentially cardiovascular and metabolic disorders, have been shown to be associated with COPD [37] and linked to an increased risk of mortality $[14,38]$. Among these comorbidities, liver diseases are hugely underreported and certainly underestimated. Divo et al. [14] prospectively followed 1664 patients with COPD for a median of 51 months with a systematic record of 79 comorbidities. Liver cirrhosis was reported in $2.5 \%$ of patients and associated with two-fold higher mortality. While liver cirrhosis was attributed to alcohol and smoking, it might originate from the progression of steatosis to cirrhosis in the context of the metabolic syndrome associated with COPD. Using a system network analysis comparing 1969 COPD and 316 non-COPD patients it was demonstrated that comorbidities encountered in COPD patients are interlinked [39]. This was the case for hepatitis, liver cirrhosis and liver cancer, and again the suggested mechanism was linked to substance abuse. As stated by the authors, such a network analysis provides information about common risk factors or disease mechanisms that are difficult to discern when studied individually. We suggest that this cluster of liver diseases is more likely to be attributable to components of metabolic syndrome and a general reduction in physical activity. The limitation of the two studies by Divo et al. [14,39], as acknowledged by
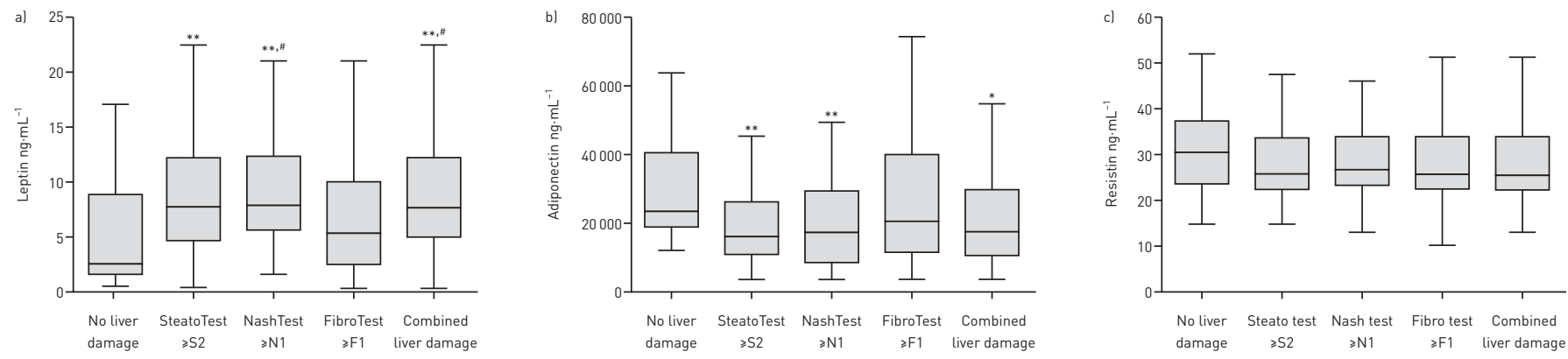

FIGURE 1 Adipokines a) leptin, b) adiponectin and c) resistin in chronic obstructive pulmonary disease patients with different types of liver damage. *: $p<0.05$ and ${ }^{* *}: p<0.01$ by Mann-Whitney test in an unadjusted model; ${ }^{*}: p<0.05$ in a logistic regression model adjusted for age, sex and body mass index.
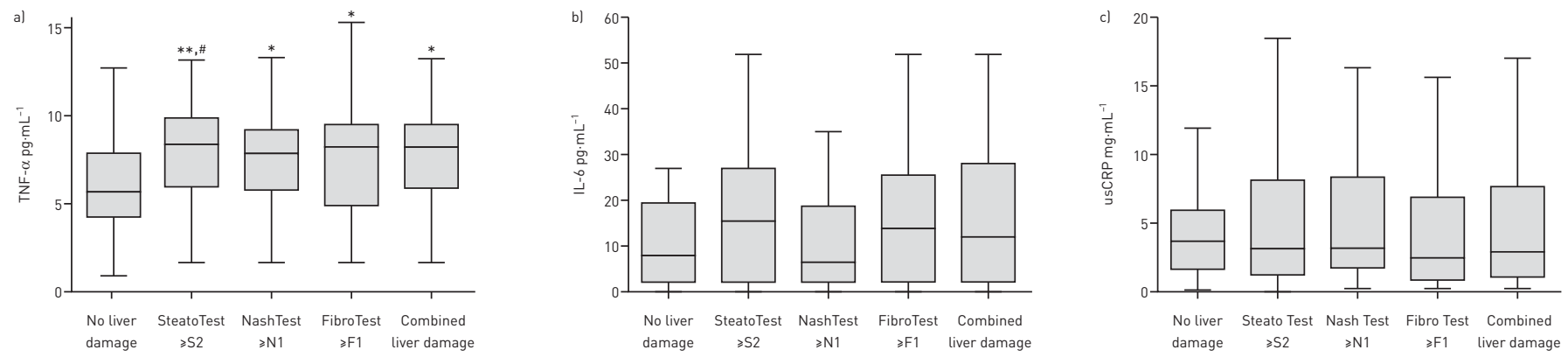

FIGURE 2 Inflammatory parameters a) tumour necrosis factor (TNF)- $\alpha$; b) interleukin (IL)-6; and c) ultrasensitive C-reactive protein (usCRP), in chronic obstructive pulmonary disease patients with different types of liver damage. *: $p<0.05$ and ${ }^{* *}$ : $p<0.01$ by Mann-Whitney test in an unadjusted model; ${ }^{\#}$ : $p<0.05$ in a logistic regression model adjusted for age, sex and body mass index. 
the authors themselves, was that the ascertainment of comorbidities was obtained by questioning the patient and from medical records. This is a sensitive method for capturing a large number of comorbidities, but lacks objective measures that would improve specificity [19] and allow grading of severity. The strengths of our study were to focus on NAFLD and exclude other liver diseases with other aetiologies and to quantify NAFLD-related alterations and the severity of liver disease using biological markers with established cut-offs that have been validated against liver biopsies.

Among its limitations, our study did not include a control group to allow a comparison between the prevalence rate in the general population and our COPD patients. Prevalence in the general population has been established through a meta-analysis reporting a combination of imaging (ultrasound, computed tomography scanning and magnetic resonance imaging/spectroscopy), liver biopsy and blood testing (elevated liver enzymes or fatty liver index, etc.) data [2].

We did not perform liver biopsy, the gold standard for confirming NAFLD diagnosis and providing prognostic information. However, it is an invasive and costly procedure, prone to minor side-effects such as pain, or more severe complications, including a risk of death of $0.03 \%$ [29]. Moreover, intra- and interobserver variabilities exist [40]. Thus, several advantages of these noninvasive tests should be stressed: compared to biopsy, they are cheaper, repeatable and lead to less interobserver variability, severe adverse events, refusal and bias [29].

Noninvasive diagnosis and quantification of NAFLD rely mainly on biomarkers or imaging techniques [7]. Both strategies have limitations and lack sensitivity or specificity. As liver biopsies were not appropriate in our clinical research setting, we decided to use FibroMax, which represents one of the best compromises for noninvasive assessment of NAFLD, having been validated against liver biopsies [29] and which is now proposed by European guidelines to screen noninvasively for NAFLD [7]. In future studies of COPD, the combination of biomarkers/scores and transient elastography with liver ultrasonography (not performed in the current study) might confer additional diagnostic accuracy. Indeed, liver ultrasound is a first-line imaging technique used to identify hepatic steatosis in patients with NAFLD [3, 31, 32] and which plays a major role in the early diagnosis of hepatocellular carcinoma in at-risk populations. The strong prevalence of steatosis, NASH and fibrosis observed in our study needs to be replicated in different COPD cohorts and using different diagnostic tools. In addition, this prevalence needs to be compared with that of the general population (with age- and smoking-matched controls) using the same noninvasive tests.

Many factors such as insulin resistance, low-grade inflammation, oxidative stress and lipotoxicity are involved in the onset and progression of NAFLD. It appears that COPD and NAFLD share common intermediary mechanisms. Our analyses showed an independent role of BMI and insulin resistance in the development of NAFLD in COPD patients. Hepatic steatosis develops in the context of increased triglyceride flux into the liver from visceral adipose tissue [41]. Furthermore, fat inflammation, which has been demonstrated in COPD [42], drives the development of NAFLD. Excessive and inflamed visceral fat is associated with circulating cytokines including leptin and TNF- $\alpha[43,44]$. We found higher leptin levels in COPD patients with NASH or with a combination of liver lesions compared to COPD patients without liver damage. A continuous dynamic cross-talk between adipokines with a positive or negative impact on NAFLD resulting in an overall beneficial or detrimental effect has been described [45]. TNF- $\alpha$ might play an initial role in the occurrence of steatosis, while leptin is known to exert a pro-steatotic and-pro fibrotic action $[45,46]$. TNF- $\alpha$ levels were significantly higher in our COPD patients with steatosis compared to those with no liver injury. TNF- $\alpha$ plays a crucial role in human and animal NAFLD [47]. Rosiglitazone has been shown to lower adipose tissue inflammation with a reduction in plasma leptin and TNF- $\alpha$ levels and less severe NAFLD [48]. The role of chronic intermittent hypoxia (CIH) in the development of NAFLD (for review see [9]) is clearly established both in rodent models and in sleep apnoea patients. In mice, $\mathrm{CIH}$ increases the expression of key lipogenic, inflammatory and fibrotic transcription factors [49]. In line with this, in our COPD cohort which underwent systematic sleep studies, untreated OSA was significantly associated with steatosis and fibrosis. Finally, low physical activity is a major determinant of NAFLD [50] and might play a key role in COPD. However, we did not objectively measure physical activity in our study and further studies are required to in order to provide evidence for this highly probable link.

\section{Conclusion and perspectives}

Owing to its deleterious consequences and independent impact on mortality [2], NAFLD should be included among COPD comorbidities. Insulin resistance, systemic inflammation and $\mathrm{CIH}$ seem to play a role in the development of NAFLD in COPD patients. Biomarkers of liver damage may allow specific COPD endotypes to be detected, leading to personalised treatments [36]. As physical activity is the first-line treatment of NAFLD [50-52], pulmonary rehabilitation could be expected to have a positive effect on NAFLD in COPD patients. In the obese inflamed COPD phenotype, lifestyle intervention with cautious weight loss might also improve NAFLD. 


\section{Acknowledgements}

The authors are grateful to Marion Perrin (INSERM U1042, HP2 Laboratory, Grenoble Alps University Hospital, Grenoble, France) for statistical analyses, Candice Trocmé (Biochemistry, Toxicology and Pharmacology Dept, Grenoble Alps University Hospital) for biochemical analyses and Alison Foote (Grenoble Alps University Hospital) for English editing. J-L. Pépin acts as guarantor for the study and is accountable for all aspects of the work and ensures that questions related to the accuracy or integrity of any part of the work are appropriately investigated and resolved.

Authors' contributions: conception and design: D. Viglino, I. Jullian-Desayes, R. Tamisier, M. Joyeux-Faure and J-L. Pépin; data acquisition, analysis and interpretation: all the authors; drafting and revising the manuscript for important intellectual content: all the authors, with a major contribution by J-L. Pépin as principal investigator. All authors read and approved the final version of the manuscript.

\section{References}

1 Chalasani N, Younossi Z, Lavine JE, et al. The diagnosis and management of non-alcoholic fatty liver disease: practice Guideline by the American Association for the Study of Liver Diseases, American College of Gastroenterology, and the American Gastroenterological Association. Hepatology 2012; 55: 2005-2023.

2 Younossi $\mathrm{ZM}$, Koenig $\mathrm{AB}$, Abdelatif $\mathrm{D}$, et al. Global epidemiology of nonalcoholic fatty liver disease meta-analytic assessment of prevalence, incidence, and outcomes. Hepatology 2016; 64: 73-84.

3 Nascimbeni F, Pais R, Bellentani S, et al. From NAFLD in clinical practice to answers from guidelines. $J$ Hepatol 2013; 59: 859-871.

4 Bedossa P, Tordjman J, Aron-Wisnewsky J, et al. Systematic review of bariatric surgery liver biopsies clarifies the natural history of liver disease in patients with severe obesity. Gut 2016 [In press DOI: 10.1136/ gutinl-2016-312238].

5 Bedossa P, Poitou C, Veyrie N, et al. Histopathological algorithm and scoring system for evaluation of liver lesions in morbidly obese patients. Hepatology 2012; 56: 1751-1759.

6 Rinella ME. Nonalcoholic fatty liver disease: a systematic review. JAMA 2015; 313: 2263-2273.

7 European Association for the Study of the Liver (EASL), European Association for the Study of Diabetes (EASD), European Association for the Study of Obesity (EASO). EASL-EASD-EASO Clinical Practice Guidelines for the management of non-alcoholic fatty liver disease. J Hepatol 2016; 64: 1388-1402.

8 Cohen JC, Horton JD, Hobbs HH. Human fatty liver disease: old questions and new insights. Science 2011; 332: 1519-1523.

9 Aron-Wisnewsky J, Clement K, Pépin J-L. Nonalcoholic fatty liver disease and obstructive sleep apnea. Metabolism 2016; 65: 1124-1135.

10 Lonardo A, Ballestri S, Marchesini G, et al. Nonalcoholic fatty liver disease: a precursor of the metabolic syndrome. Dig Liver Dis 2015; 47: 181-190.

11 Ballestri S, Nascimbeni F, Romagnoli D, et al. The independent predictors of non-alcoholic steatohepatitis and its individual histological features: insulin resistance, serum uric acid, metabolic syndrome, alanine aminotransferase and serum total cholesterol are a clue to pathogenesis and candidate targets for treatment. Hepatol Res 2016; 46: 1074-1087.

12 Ballestri S, Zona S, Targher G, et al. Nonalcoholic fatty liver disease is associated with an almost twofold increased risk of incident type 2 diabetes and metabolic syndrome. Evidence from a systematic review and meta-analysis. J Gastroenterol Hepatol 2016; 31: 936-944.

13 Targher G, Byrne CD, Lonardo A, et al. Non-alcoholic fatty liver disease and risk of incident cardiovascular disease: a meta-analysis. J Hepatol 2016; 65: 589-600.

14 Divo M, Cote C, de Torres JP, et al. Comorbidities and risk of mortality in patients with chronic obstructive pulmonary disease. Am J Respir Crit Care Med 2012; 186: 155-161.

15 Cebron Lipovec N, Beijers RJ, van den Borst B, et al. The prevalence of metabolic syndrome in chronic obstructive pulmonary disease: a systematic review. COPD 2016; 13: 399-406.

16 Sin DD, MacNee W. Chronic obstructive pulmonary disease and cardiovascular diseases: a "vulnerable" relationship. Am J Respir Crit Care Med 2013; 187: 2-4.

17 Van Eeden S, Leipsic J, Paul Man SF, et al. The relationship between lung inflammation and cardiovascular disease. Am J Respir Crit Care Med 2012; 186: 11-16.

18 Marquis $\mathrm{K}$, Maltais $\mathrm{F}$, Duguay V, et al. The metabolic syndrome in patients with chronic obstructive pulmonary disease. J Cardiopulm Rehabil 2005; 25: 226-232.

19 Vanfleteren LE. Does COPD stand for "COmorbidity with Pulmonary Disease"? Eur Respir J 2015; 45: 14-17.

20 Fabbri LM, Luppi F, Beghé B, et al. Complex chronic comorbidities of COPD. Eur Respir J 2008; 31: 204-212.

21 Tkacova R. Systemic inflammation in chronic obstructive pulmonary disease: may adipose tissue play a role? Review of the literature and future perspectives. Mediators Inflamm 2010; 2010: 585989.

22 Chen W, Thomas J, Sadatsafavi M, et al. Risk of cardiovascular comorbidity in patients with chronic obstructive pulmonary disease: a systematic review and meta-analysis. Lancet Respir Med 2015; 3: 631-639.

23 Cleutjens F, Triest F, Wilke S, et al. New insights in chronic obstructive pulmonary disease and comorbidity. Am J Respir Crit Care Med 2015; 191: 1081-1082.

24 van den Borst B, Gosker HR, Schols AM. Central fat and peripheral muscle: partners in crime in chronic obstructive pulmonary disease. Am J Respir Crit Care Med 2013; 187: 8-13.

25 Minakata Y, Ueda H, Akamatsu K, et al. High COPD prevalence in patients with liver disease. Intern Med 2010; 49: 2687-2691.

26 Cancello R, Tordjman J, Poitou C, et al. Increased infiltration of macrophages in omental adipose tissue is associated with marked hepatic lesions in morbid human obesity. Diabetes 2006; 55: 1554-1561.

27 Minville C, Hilleret M-N, Tamisier R, et al. Nonalcoholic fatty liver disease, nocturnal hypoxia, and endothelial function in patients with sleep apnea. Chest 2014; 145: 525-533.

28 Aron-Wisnewsky J, Minville C, Tordjman J, et al. Chronic intermittent hypoxia is a major trigger for non-alcoholic fatty liver disease in morbid obese. J Hepatol 2012; 56: 225-233.

29 Munteanu M, Ratziu V, Morra R, et al. Noninvasive biomarkers for the screening of fibrosis, steatosis and steatohepatitis in patients with metabolic risk factors: FibroTest-FibroMax experience. J Gastrointest Liver Dis 2008; 17: $187-191$. 
30 Antuna-Puente B, Disse E, Rabasa-Lhoret R, et al. How can we measure insulin sensitivity/resistance? Diabetes Metab 2011; 37: 179-188.

31 Ballestri S, Romagnoli D, Nascimbeni F, et al. Role of ultrasound in the diagnosis and treatment of nonalcoholic fatty liver disease and its complications. Expert Rev Gastroenterol Hepatol 2015; 9: 603-627.

32 Loria P, Adinolfi LE, Bellentani S, et al. Practice guidelines for the diagnosis and management of nonalcoholic fatty liver disease. A decalogue from the Italian Association for the Study of the Liver (AISF) Expert Committee. Dig Liver Dis 2010; 42: 272-282.

33 Non-alcoholic Fatty Liver Disease Study Group, Lonardo A, Bellentani S, et al. Epidemiological modifiers of non-alcoholic fatty liver disease: focus on high-risk groups. Dig Liver Dis 2015; 47: 997-1006.

34 Morra R, Munteanu M, Imbert-Bismut F, et al. FibroMAX: towards a new universal biomarker of liver disease? Expert Rev Mol Diagn 2007; 7: 481-490.

35 Bedossa P, Poynard T. An algorithm for the grading of activity in chronic hepatitis C. The METAVIR Cooperative Study Group. Hepatology 1996; 24: 289-293.

36 Vanfleteren LE, Spruit MA, Wouters EFM, et al. Management of chronic obstructive pulmonary disease beyond the lungs. Lancet Respir Med 2016; 4: 911-924.

37 Vanfleteren LE, Spruit MA, Groenen M, et al. Clusters of comorbidities based on validated objective measurements and systemic inflammation in patients with chronic obstructive pulmonary disease. Am $J$ Respir Crit Care Med 2013; 187: 728-735.

38 Rennard SI, Locantore N, Delafont B, et al. Identification of five chronic obstructive pulmonary disease subgroups with different prognoses in the ECLIPSE cohort using cluster analysis. Ann Am Thorac Soc 2015; 12: 303-312.

39 Divo MJ, Casanova C, Marin JM, et al. COPD comorbidities network. Eur Respir J 2015; 46: 640-650.

40 Ratziu V, Charlotte F, Heurtier A, et al. Sampling variability of liver biopsy in nonalcoholic fatty liver disease. Gastroenterology 2005; 128: 1898-1906.

41 Hashimoto E, Taniai M, Tokushige K. Characteristics and diagnosis of NAFLD/NASH. J Gastroenterol Hepatol 2013; 28: Suppl. 4, 64-70.

42 Vanfleteren LE, van Meerendonk AM, Franssen FM, et al. A possible link between increased metabolic activity of fat tissue and aortic wall inflammation in subjects with COPD. A retrospective ${ }^{18}$ F-FDG-PET/CT pilot study. Respir Med 2014; 108: 883-890.

43 Rutten EPA, Breyer MK, Spruit MA, et al. Abdominal fat mass contributes to the systemic inflammation in chronic obstructive pulmonary disease. Clin Nutr 2010; 29: 756-760.

44 van den Borst B, Gosker HR, Koster A, et al. The influence of abdominal visceral fat on inflammatory pathways and mortality risk in obstructive lung disease. Am J Clin Nutr 2012; 96: 516-526.

45 Polyzos SA, Kountouras J, Mantzoros CS. Adipokines in nonalcoholic fatty liver disease. Metabolism 2016; 65: 1062-1079.

46 Polyzos SA, Mantzoros CS. Nonalcoholic fatty future disease. Metabolism 2016; 65: 1007-1016.

47 Tilg H. The role of cytokines in non-alcoholic fatty liver disease. Dig Dis 2010; 28: 179-185.

48 Mulder P, Morrison MC, Verschuren L, et al. Reduction of obesity-associated white adipose tissue inflammation by rosiglitazone is associated with reduced non-alcoholic fatty liver disease in LDLr-deficient mice. Sci Rep 2016; 6: 31542 .

49 Yao Q, Shin M-K, Jun JC, et al. Effect of chronic intermittent hypoxia on triglyceride uptake in different tissues. J Lipid Res 2013; 54: 1058-1065.

50 Lassailly G, Caiazzo R, Pattou F, et al. Perspectives on treatment for nonalcoholic steatohepatitis. Gastroenterology 2016; 150: 1835-1848.

51 Zhang X, Tong M-M, Zhang M-Z, et al. Risk factors of nosocomial bloodstream infections in surgical intensive care unit. Int J Clin Exp Med 2015; 8: 16682-16687.

52 Prenner S, Rinella ME. Moderate exercise for nonalcoholic fatty liver disease. JAMA Intern Med 2016; 176: 1083-1084. 